\title{
Blow-up criteria for a two-fluid model of the truncated Euler equations
}

\author{
Yonglin $\mathrm{Xu}^{1 *}$, Haizhou $\mathrm{MaO}^{2}$, Xiaohong Fan ${ }^{3}$ and Liqiang $\mathrm{Ma}^{3 *}$
}

\author{
"Correspondence: \\ yonglinxu04@163.com; \\ liqiangma1234@126.com \\ 1 Mathematics and Computer \\ College, North-western Minorities \\ University, Lanzhou, Gansu 730030, \\ China \\ ${ }^{3}$ Foundation Department, \\ Shandong Transport Vocational \\ College, Weifang, Shandong \\ 261206, China \\ Full list of author information is \\ available at the end of the article
}

\begin{abstract}
In this paper, we study a two-fluid model of the truncated Euler equations with partial viscosity. We obtain new blow-up criteria for a solution of the system in terms of vorticity in the homogeneous Besov space $\dot{B}_{\infty, \infty}^{-1}$.
\end{abstract}

Keywords: blow-up criterion; truncated Euler equations; Besov space

\section{Introduction and main results}

In this paper, we are concerned with the regularity of the following two-fluid model of the truncated Euler equations with partial viscosity:

$$
\left\{\begin{array}{l}
u_{t}-\Delta u+u \cdot \nabla u+\nabla p=\theta e_{3}, \quad(x ; t) \in \mathbb{R}^{3} \times(0 ; 1), \\
\theta_{t}+u \cdot \nabla \theta=|\nabla u|^{2}, \\
\nabla \cdot u=0, \\
u(x, 0)=u_{0}, \quad \theta(x, 0)=\theta_{0},
\end{array}\right.
$$

where $u=\left(u^{1}(x, t), u^{2}(x, t), u^{3}(x, t)\right)$ denotes the fluid velocity vector field, $p=p(x, t)$ is the scalar pressure, $e_{3}=(0,0,1)^{T}$, while $u_{0}$ and $\theta_{0}$ are given initial velocity and initial temperature, respectively, with $\nabla \cdot u_{0}=0$. For more detailed background of the system, we refer the readers to [1] and the references therein. When $\theta=0$, the system reduces to the well-known Navier-Stokes equations.

It is well known that the question of global existence or finite time blow-up of smooth solutions for the 3D incompressible Navier-Stokes equations is still unsolved. This challenging problem has attracted significant attention. Therefore, it is interesting to study the blow-up criterion of the solutions for system (1.1). Recently, Sun and Fan [2] first proved the following blow-up criterion for system (1.1):

$$
\begin{aligned}
& \operatorname{curl} u \in L^{1}\left(0, T ; \dot{B}_{\infty, \infty}^{0}\left(\mathbb{R}^{3}\right)\right), \\
& u \in L^{2}\left(0, T ; \dot{B}_{\infty, \infty}^{0}\left(\mathbb{R}^{3}\right)\right) .
\end{aligned}
$$

Our purpose in this paper is to establish blow-up criteria of a strong solution for the two-fluid model of the truncated Euler equations in terms of vorticity in the homogeneous Besov space $\dot{B}_{\infty, \infty}^{-1}$. The main difficulty is without viscosity in the second equation for system (1.1).

Now we state our main results as follows.

@2013 Xu et al.; licensee Springer. This is an Open Access article distributed under the terms of the Creative Commons Attribution License (http://creativecommons.org/licenses/by/2.0), which permits unrestricted use, distribution, and reproduction in any medium, provided the original work is properly cited. 
Theorem 1.1 Let $\left(u_{0}, \theta_{0}\right) \in H^{2}\left(\mathbb{R}^{3}\right),(u(\cdot, t), \theta(\cdot, t))$ be a strong solution to system (1.1) with the initial data $\left(u_{0}, \theta_{0}\right)$ for $0 \leq t<T$. If the solution $u$ satisfies the following condition:

$$
\operatorname{curl} u \in L^{2}\left(0, T ; \dot{B}_{\infty, \infty}^{-1}\right)
$$

then the solution $(u, \theta)$ can be extended smoothly beyond $t=T$.

\section{Preliminaries and lemmas}

Throughout this paper, we introduce some function spaces, notations and important inequalities.

Let $e^{t \Delta}$ denote the heat semigroup defined by

$$
e^{t \Delta} f=K_{t} * f, \quad K_{t}=(4 \pi t)^{-\frac{3}{2}} \exp \left(-\frac{|x|^{2}}{4 t}\right)
$$

for $t>0$ and $x \in \mathbb{R}^{3}$, where $*$ denotes the convolution of functions defined on $\mathbb{R}^{3}$.

We now recall the definition of the homogeneous Besov space with negative indices $\dot{B}_{\infty, \infty}^{-\alpha}$ on $\mathbb{R}^{3}$ and the homogeneous Sobolev space $\dot{H}_{q}^{\alpha}$ of exponent $\alpha>0$. It is known (p.192 of Ref. [3]) that $f \in \mathcal{S}^{\prime}\left(\mathbb{R}^{3}\right)$ belongs to $\dot{B}_{\infty, \infty}^{-\alpha}$ if and only if $e^{t \Delta} f \in L^{\infty}$ for all $t>0$ and $t^{\frac{\alpha}{2}}\left\|e^{t \Delta} f\right\|_{\infty} \in L^{\infty}\left(0, \infty ; L^{\infty}\right)$. The norm of $\dot{B}_{\infty, \infty}^{-\alpha}$ is defined, up to equivalence, by

$$
\|f\|_{\dot{B}_{\infty, \infty}^{-\alpha}}=\sup _{t>0}\left(t^{\frac{\alpha}{2}}\left\|e^{t \Delta} f\right\|_{\infty}\right)
$$

We introduce now the homogeneous Sobolev space $\dot{H}_{q}^{\alpha}\left(\mathbb{R}^{3}\right)$, which is defined as the set of functions $f \in L^{r}\left(\mathbb{R}^{3}\right), \frac{1}{r}=\frac{1}{q}-\frac{\alpha}{3}$ such that $(-\Delta)^{\frac{\alpha}{2}} f \in L^{q}\left(\mathbb{R}^{3}\right)$. This space is endowed with the norm

$$
\|f\|_{\dot{H}_{q}^{\alpha}}=\left\|(-\Delta)^{\frac{\alpha}{2}} f\right\|_{L^{q}},
$$

and when $q=2$, we just let $\dot{H}_{2}^{\alpha}\left(\mathbb{R}^{3}\right)=\dot{H}^{\alpha}\left(\mathbb{R}^{3}\right)$.

Lemma 2.1 [4] Let $1<p<q<\infty$ and $s=\alpha\left(\frac{q}{p}-1\right)>0$. Then there exists a constant depending only on $\alpha, p$ and $q$ such that the estimate

$$
\|f\|_{L^{q}} \leq C\left\|(-\Delta)^{\frac{s}{2}} f\right\|_{L^{p}}^{\frac{p}{q}}\|f\|_{\dot{B}_{\infty, \infty}^{-\alpha}}^{1-\frac{p}{q}}
$$

holds for all $f \in \dot{H}_{p}^{\alpha}\left(\mathbb{R}^{3}\right) \cap \dot{B}_{\infty, \infty}^{-\alpha}\left(\mathbb{R}^{3}\right)$.

In particular, for $s=1, p=2$ and $q=4$, we get $\alpha=1$ and

$$
\|f\|_{L^{4}} \leq C\|f\|_{\dot{H}^{1}}^{\frac{1}{2}}\|f\|_{\dot{B}_{\infty, \infty}^{-1}}^{\frac{1}{2}} .
$$

Lemma 2.2 [5] For any function $f \in W^{1, s}\left(\mathbb{R}^{3}\right)(s \geq 1)$, and $r \geq 1$, we have

$$
\|f\|_{L^{\gamma}} \leq C\|f\|_{L^{2}}^{1-\alpha}\|\nabla f\|_{L^{2}}^{\alpha}
$$


where

$$
\alpha=\frac{\frac{1}{r}-\frac{1}{\gamma}}{\frac{1}{3}-\frac{1}{s}-\frac{1}{r}}
$$

and $C$ is a constant independent off.

\section{Proof of the main results}

In this section, we prove Theorem 1.1.

Proof of Theorem 1.1 First, we multiply the both sides of the first equation of (1.1) by $u$ and the second equation of (1.1) by $\theta$, respectively; after integration by parts over $\mathbb{R}^{3}$, we get

$$
\begin{aligned}
& \frac{1}{2} \frac{d}{d t} \int u^{2} d x+\int|\nabla u|^{2} d x \leq \int|u \theta| d x, \\
& \frac{1}{2} \frac{d}{d t} \int \theta^{2} d x=\int \theta|\nabla u|^{2} d x .
\end{aligned}
$$

Next, we multiply the both sides of the first equation of $(1.1)$ by $(-\Delta u)$; after integration by parts over $\mathbb{R}^{3}$, we get

$$
\frac{1}{2} \frac{d}{d t} \int|\nabla u|^{2} d x+\int|\Delta u|^{2} d x \leq \int u \cdot \nabla u \Delta u d x+\int|\Delta u| \theta d x .
$$

Using the Young inequality and adding (3.2) to (3.3), we obtain

$$
\begin{aligned}
& \frac{d}{d t}\left(\int|\nabla u|^{2} d x+\int \theta^{2} d x\right)+\int|\Delta u|^{2} d x \\
& \quad \leq \int u \cdot \nabla u \Delta u d x+\int \theta|\nabla u|^{2} d x+\int \theta^{2} d x .
\end{aligned}
$$

It is easy to see that

$$
\begin{aligned}
\int u \cdot \nabla u \Delta u d x & =-\sum_{i, k} \int \partial_{k} u \cdot \partial_{i} u \cdot \partial_{k} u d x \\
& \leq\|\nabla u\|_{L^{2}}\|\nabla u \nabla u\|_{L^{2}} \\
& \leq\|\nabla u\|_{L^{2}}\|\nabla u\|_{L^{4}}^{2} \\
& \leq\|\nabla u\|_{L^{2}}\|\nabla u\|_{\dot{B}_{\infty, \infty}^{-1}}\|\Delta u\|_{L^{2}} \\
& \leq C\|\nabla u\|_{L^{2}}^{2}\|\nabla \times u\|_{\dot{B}_{\infty, \infty}^{-1}}^{2}+\frac{1}{2}\|\Delta u\|_{L^{2}}^{2},
\end{aligned}
$$

where we use the Hölder inequality, the Young inequality and (2.2).

Similarly,

$$
\begin{aligned}
\int \theta|\nabla u|^{2} d x & \leq\|\theta\|_{L^{2}}\|\nabla u \nabla u\|_{L^{2}} \\
& \leq\|\theta\|_{L^{2}}\|\nabla u\|_{L^{4}}^{2}
\end{aligned}
$$




$$
\begin{aligned}
& \leq\|\theta\|_{L^{2}}\|\nabla u\|_{\dot{B}_{\infty, \infty}^{-1}}\|\Delta u\|_{L^{2}} \\
& \leq C\|\theta\|_{L^{2}}^{2}\|\nabla \times u\|_{\dot{B}_{\infty, \infty}^{-1}}^{2}+\frac{1}{2}\|\Delta u\|_{L^{2}}^{2} .
\end{aligned}
$$

Putting (3.5) and (3.6) into (3.4) and applying the Gronwall inequality yields

$$
u \in L^{\infty}\left(0, T ; H^{1}\right) \cap L^{2}\left(0, T ; H^{2}\right), \quad \theta \in L^{\infty}\left(0, T ; L^{2}\right) .
$$

Multiplying the both sides of the second equation of (1.1) by $|\theta|^{p-2} \theta(2<p<\infty)$, after integration by parts over $\mathbb{R}^{3}$, we deduce that

$$
\frac{1}{p} \frac{d}{d t} \int \theta^{p} d x \leq \int|\theta|^{p-1}|\nabla u|^{2} d x \leq\|\theta\|_{L^{p}}^{p-1}\|\nabla u\|_{L^{2 p}}^{2} \leq\|\theta\|_{L^{p}}^{p-1}\|u\|_{H^{2}}^{2},
$$

which implies that

$$
\theta \in L^{\infty}\left(0, T ; L^{p}\right) \quad(2<p<\infty)
$$

Taking curl on the both sides of the first equation of (1.1), we obtain the following vorticity formulation for the vorticity field $\omega=\operatorname{curl} u$ :

$$
\partial_{t} \omega+u \cdot \nabla \omega-\Delta \omega=\operatorname{curl}\left(\theta e_{3}\right)+\omega \cdot \nabla u
$$

and then multiplying the both sides of the vorticity equation by $|\omega|^{p-2} \omega(p>2)$, after integration by parts over $\mathbb{R}^{3}$, we infer that

$$
\begin{aligned}
\frac{d}{d t} \int v^{2} d x+\int|\nabla v|^{2} d x & \leq\left.\left. C \int|\theta| \nabla|| \omega\right|^{p-2} \omega\left|d x+C \int \nabla u\right| \omega\right|^{p} d x \\
& \leq C \int|\theta \| v|^{\frac{p-2}{p}}|\nabla v| d x+C \int \nabla u|\omega|^{\frac{p}{2}}|\omega|^{\frac{p}{2}} d x \\
& \leq C\|\theta\|_{L^{\frac{4 p}{p+2}}}\|v\|_{L^{4}}^{1-\frac{2}{p}}\|\nabla v\|_{L^{2}}+C\|\nabla u\|_{L^{2}}\|v\|_{L^{4}}^{2} \\
& \leq C\|v\|_{L^{4}}^{1-\frac{2}{p}}\|\nabla v\|_{L^{2}}+C\|v\|_{L^{2}}^{\frac{1}{2}}\|\nabla v\|_{L^{2}}^{\frac{3}{2}} \\
& \leq C\|v\|_{L^{2}}^{\frac{1}{2}-\frac{1}{p}}\|\nabla v\|_{L^{2}}^{\frac{3}{2}-\frac{1}{p}}+C\|v\|_{L^{2}}^{\frac{1}{2}}\|\nabla v\|_{L^{2}}^{\frac{3}{2}} \\
& \leq C\|v\|_{L^{2}}^{2}+\frac{1}{2}\|\nabla v\|_{L^{2}}^{2}
\end{aligned}
$$

where $v:=|\omega|^{\frac{p}{2}}$. Using the Gronwall inequality, we obtain

$$
\omega \in L^{\infty}\left(0, T ; L^{p}\right) \quad(p>2)
$$

which implies that

$$
\nabla u \in L^{\infty}\left(0, T ; L^{p}\right) \quad(p>2) .
$$


On the other hand, since $u$ is a solution of the Stokes system

$$
u_{t}-\Delta u+\nabla p=-u \cdot \nabla u+\theta e_{3} \in L^{\infty}\left(0, T ; L^{p}\right)
$$

it follows from the $W^{2, p}$-theory of the Stokes system that

$$
u \in L^{p}\left(0, T ; W^{2, p}\right) \quad(p>2) .
$$

So,

$$
\nabla u \in L^{p}\left(0, T ; L^{\infty}\right) \quad(p>2) .
$$

Differentiating the second equation of (1.1) with respect to $x_{i}(1 \leq i \leq 3)$ and multiplying the resulting equation by $\partial_{i} \theta$, and then integrating by parts over $\mathbb{R}^{3}$, we get

$$
\begin{aligned}
& \frac{1}{2} \frac{d}{d t} \int|\nabla \theta|^{2} d x \leq C \int|\nabla u||\nabla \theta|^{2} d x+C \int|\nabla u||\Delta u||\nabla \theta| d x \\
& \leq C\|\nabla u\|_{L^{\infty}}\|\nabla \theta\|_{L^{2}}^{2}+C\|\nabla u\|_{L^{\infty}}\|\Delta u\|_{L^{2}}\|\nabla \theta\|_{L^{2}} .
\end{aligned}
$$

By (3.12) and the Gronwall inequality, we have

$$
\theta \in L^{\infty}\left(0, T ; H^{1}\right)
$$

We multiply the both sides of the equation of (3.8) by $(-\Delta \omega)$; after integration by parts over $\mathbb{R}^{3}$, we get

$$
\begin{aligned}
\frac{1}{2} \frac{d}{d t} \int|\nabla \omega|^{2} d x+\int|\Delta \omega|^{2} d x & \leq \int|u|\left|\nabla \omega\left\|\Delta \omega\left|d x+\int\right| \Delta \omega\right\| \nabla \theta\right| d x \\
& \leq\|u\|_{L^{4}}\|\Delta \omega\|_{L^{2}}\|\nabla \omega\|_{L^{4}}+\|\nabla \theta\|_{L^{2}}\|\Delta \omega\|_{L^{2}} \\
& \leq C\|\Delta \omega\|_{L^{2}}^{\frac{1}{2}}\|\nabla \omega\|_{L^{2}}^{\frac{3}{2}}+C\|\Delta \omega\|_{L^{2}} \\
& \leq C\|\nabla \omega\|_{L^{2}}^{2}+\frac{1}{2}\|\Delta \omega\|_{L^{2}}^{2}+C
\end{aligned}
$$

the Gronwall inequality yields

$$
u \in L^{\infty}\left(0, T ; H^{2}\right) \cap L^{2}\left(0, T ; H^{3}\right) .
$$

In the following calculations, we use the following commutator estimate and bilinear estimate [6]:

$$
\begin{aligned}
& \left\|\Lambda^{s}(f g)-f \Lambda^{s} g\right\|_{L^{p}} \leq\left(\|\nabla f\|_{L^{p_{1}}}\left\|\Lambda^{s-1} g\right\|_{L^{q_{1}}}+\left\|\Lambda^{s} f\right\|_{L^{p_{2}}}\|g\|_{L^{q_{2}}}\right), \\
& \left\|\Lambda^{s}(f g)\right\|_{L^{p}} \leq\left(\|f\|_{L^{p_{1}}}\left\|\Lambda^{s} g\right\|_{L^{q_{1}}}+\left\|\Lambda^{s} f\right\|_{L^{p_{2}}}\|g\|_{L^{q_{2}}}\right),
\end{aligned}
$$

with $s>0, \Lambda^{s}=(-\Delta)^{\frac{s}{2}}$ and $\frac{1}{p}=\frac{1}{p_{1}}+\frac{1}{q_{1}}=\frac{1}{p_{2}}+\frac{1}{q_{2}}$. Taking the operation $\Lambda^{2}$ on both sides of the second equation of (1.1), then multiplying them by $\Lambda^{2} \theta$, and integrating by parts over 
$\mathbb{R}^{3}$, we have

$$
\begin{aligned}
\frac{1}{2} \frac{d}{d t} \int\left|\Lambda^{2} \theta\right|^{2} d x \leq & \left|\int\left(\Lambda^{2}(u \cdot \nabla \theta)-u \nabla \Lambda^{2} \theta\right) d x\right|+\int \Lambda^{2}\left(|\nabla u|^{2}\right) \Lambda^{2} \theta d x \\
\leq & C\left(\|\nabla u\|_{L^{\infty}}\left\|\Lambda^{2} \theta\right\|_{L^{2}}+\|\| \nabla u\left\|_{L^{\infty}}\right\| \Lambda^{2} \theta\left\|_{L^{2}} u\right\|_{L^{3}}\|\nabla \theta\|_{L^{6}}\right)\left\|\Lambda^{2} \theta\right\|_{L^{2}} \\
& +C\|\nabla u\|_{L^{\infty}}\left\|\Lambda^{3} u\right\|_{L^{2}}\left\|\Lambda^{2} \theta\right\|_{L^{2}} .
\end{aligned}
$$

Combining (3.12) with (3.14) and using the Gronwall inequality, we obtain

$$
\theta \in L^{\infty}\left(0, T ; H^{2}\right)
$$

This completes the proof.

\section{Competing interests}

The authors declare that they have no competing interests.

\section{Authors' contributions}

YX provided the model in the paper and some important references [1, 2]. He performed the some analysis work. HM and XF provided the ideas about blow-up criteria in terms of vorticity in the homogeneous Besov space and participated in the design of the study. LM mainly carried out inferences and computations of formulas in the paper. All authors read and approved the final manuscript.

\section{Author details}

${ }^{1}$ Mathematics and Computer College, North-western Minorities University, Lanzhou, Gansu 730030, China. ${ }^{2}$ Foundation Department, Zhejiang Industry and Trade Vocational College, Wenzhou, Zhejiang 325000, China. ${ }^{3}$ Foundation Department, Shandong Transport Vocational College, Weifang, Shandong 261206, China.

\section{Acknowledgements}

Research supported by the National Natural Science Foundation of China (11161041), the Fundamental Research Funds for the Central Universities (No. 31920130006) and Middle-Younger Scientific Research Fund (No. 12XB39).

\section{Received: 4 March 2013 Accepted: 18 September 2013 Published: 07 Nov 2013}

\section{References}

1. Krstulovic, G, Brachet, M: Two-fluid model of the truncated Euler equations. Physica D 237, 2015-2019 (2008)

2. Sun, J, Fan, J: Regularity criterion for a two-fluid model of the truncated Euler equations. Acta Math. Sci. 30, 1693-1698 (2010) (in Chinese)

3. Triebel, H: Interpolation Theory, Function Spaces, Differential Operators. North-Holland Mathematical Library, vol. 18. North-Holland, Amsterdam (1978)

4. Meyer, Y, Gerard, P, Oru, F: Inégalités de Sobolev précisées. In: Séminaire Équations aux dérivées partielles (Polytechnique) (1996-1997), Exp. No. 4, 8p

5. Ladyzhenskaya, O, Solonnikov, V, Ural'tseva, N: Linear and Quasilinear Equations of Parabolic Type. Translated from the Russian by S. Smith. Translations of Mathematical Monographs. Amer. Math. Soc., Providence (1968)

6. Kato, T, Ponce, G: Commutator estimates and the Euler and Navier-Stokes equations. Commun. Pure Appl. Math. 41(7), 891-907 (1988)

10.1186/1029-242X-2013-461

Cite this article as: Xu et al.: Blow-up criteria for a two-fluid model of the truncated Euler equations. Journal of Inequalities and Applications 2013, 2013:461 